\title{
Neuroradiologie interaktiv: Neuer Kurs auf conrad
}

Die Abteilung für Diagnostische und interventionelle Neuroradiologie des Universitätsklinikums Tübingen unter Leitung der Ärztlichen Direktorin Prof. Dr. Ulrike Ernemann bietet seit Juli 2020 für DRG-Mitglieder einen kostenlosen, interaktiven NeuroradiologieKurs auf conrad, der digitalen Lernplattform der DRG, an.

Dieser Kurs richtet sich an Studierende sowie Kolleginnen und Kollegen mit Interesse an der Neuroradiologie. Er vermittelt Basis- wissen über das Erkennen und die Bewertung wichtiger Krankheitsbilder in der Neuroradiologie. Der Kurs bietet aktuell knapp 50 aufgearbeitete Fälle aus den Themenfeldern Traumatologie, nicht-traumatische zerebrale Blutungen, Schlaganfall, Neuroonkologie, entzündliche ZNS-Erkrankungen, Kinderneuroradiologie und Neurodegeneration. Die Vermittlung der Lerninhalte erfolgt anhand der Fälle und zusätzlich bereitgestellten Lernmaterialien. In Abhängigkeit vom Feedback der Teilnehmenden wird der Kurs zukünftig um weitere Fälle erweitert.

\section{KENNEN SIE SCHON CONRAD?}

conrad, die digitale Lernplattform der DRG, bietet ein umfangreiches Angebot an Online-Inhalten. Neben interaktiven Fallsammlungen inklusive DICOM-Datensätzen und MultipleChoice-Fragen finden Nutzerinnen und Nutzer dort auch Vorträge von Kongressen und Seminaren.

Mitglieder der DRG und der VMTB können auf eine Mehrzahl der Inhalte wie beispielsweise den neuen Neuroradiologie-Kurs kostenlos zugreifen. Weitere Informationen erhalten Sie auf www.conrad.drg.de. 


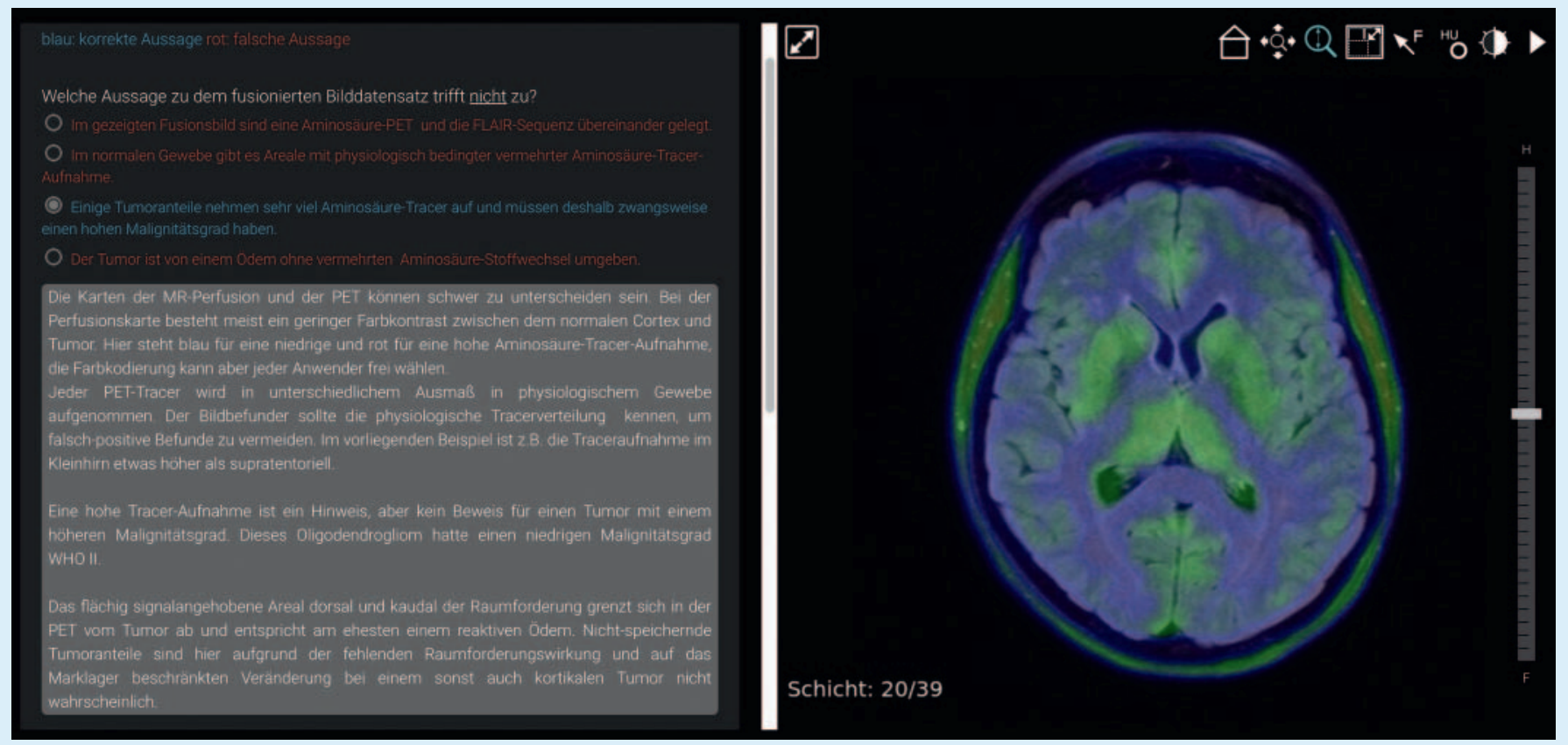

Fallbasierte Multiple-Choice-Fragen und ergänzende Erklärtexte. @ Universitätsklinikum Tübingen

\section{Tumore: Niedriggradiges Gliom}

\section{Typische Bildbefunde}
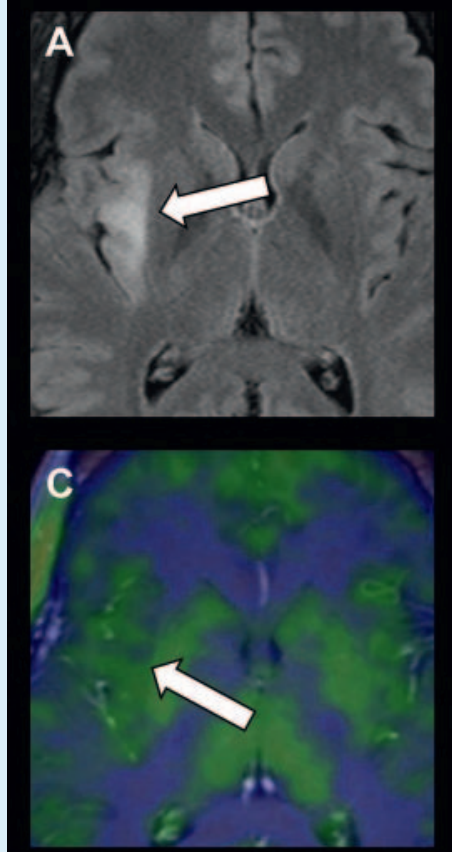

Abb. A: Hyperintenser Tumor - kann auch inhomogen sein, häufig kein oder wenig Ödem, hier Oligodendrogliom WHO Grad II rechts insulär (MRT, FLAIR-Sequenz axial)

Abb. B: Keine Schrankenstörung - fleckige oder flaue, selten auch ausgeprägte Schrankenstörungen, hier Astrozytom WHO Grad II links frontal (MRT, kontrastmittelgestützte T1-gewichtete Sequenz axial)

Abb. C: Kein vermehrter Stoffwechsel - oft keine vermehrte Aminosäure-Tracer-Aufnahme, vergleiche Gegenseite (Fusionsbild aus kontrastmittelgestütztert T1-gewichteter MR-Sequenz und Aminosäure-PET axial)

Abb. D: Keine vermehrte Durchblutung - keine erhöhte Perfusion (cerebrales Blutvolumen, CBV) im Vergleich zur Umgebung (Fusionsbild aus MRFLAIR-Sequenz und kontrastmittelgestützter CBVPerfusionskarte axial)
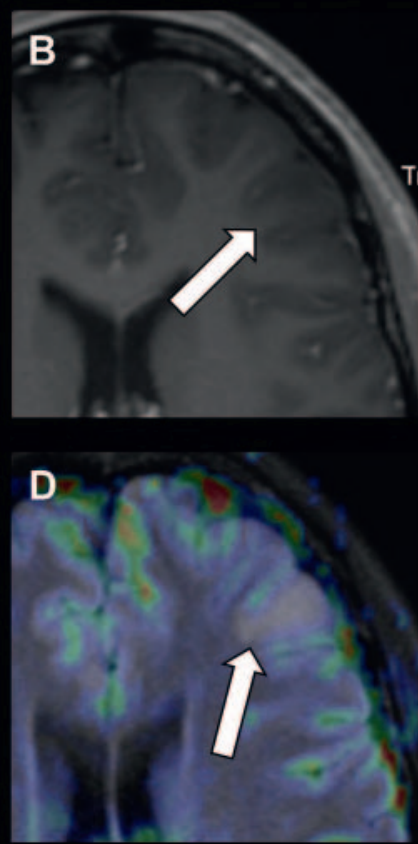

Fallbasierte Lernmaterialien am Beispiel des niedriggradigen Glioms. @ Universitätsklinikum Tübingen 


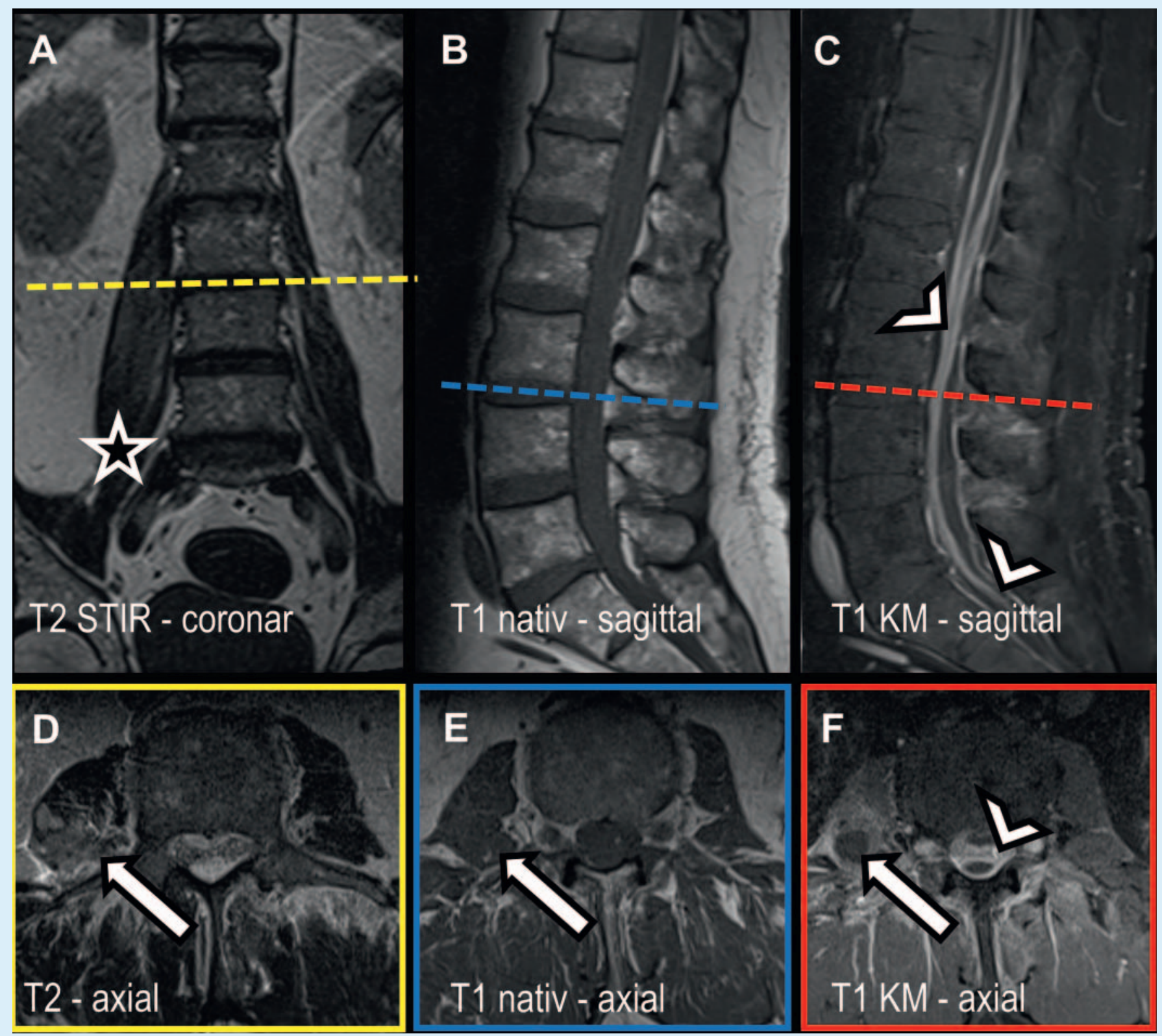

SMRT bei einem 68-jährigen Patienten mit Meningitis. Die native (A, B, D, E) und sekundär KM-angehobene MRT der Lendenwirbelsäule (C, F) zeigt in der coronaren T2 gewichteten STIR im Seitenvergleich eine deutliche Schwellung und T2 hyperintenses Ödem im rechten Musculus psoas (A, Stern), die axiale T2, native T1 und KM-angehobene T1 auf Höhe des Bandscheibenfachs LWK 3/4 (A-C, gestrichelte Linie) zeigen abszedierte Herde mit typischer Einschmelzung und randlicher KM-Mehraufnahme im rechten Musculus psoas (D-F Pfeile). Ausgeprägte epidurale Flüssigkeitsdepots mit kräftiger KM-Anreicherung entlang des gesamten Spinalkanals bis ins distale Sakrum mit Punctum maximum in der distalen LWS bzw. lumbosakralen Übergang. Eine deutliche Pelottierung des Duralsacks entlang des gesamten Spinalkanals, partiell mit höhergradiger Spinalkanalstenose, ist zu erkennen (C, F, Pfeilspitzen). Die Wirbelkörper und Bandscheibenfächer zeigen keine umschriebene pathologische Signalsteigerung. Der Befund passt zu einer ausgeprägten epiduralen Abszedierung entlang des gesamten Spinalkanals bei Meningitis. 


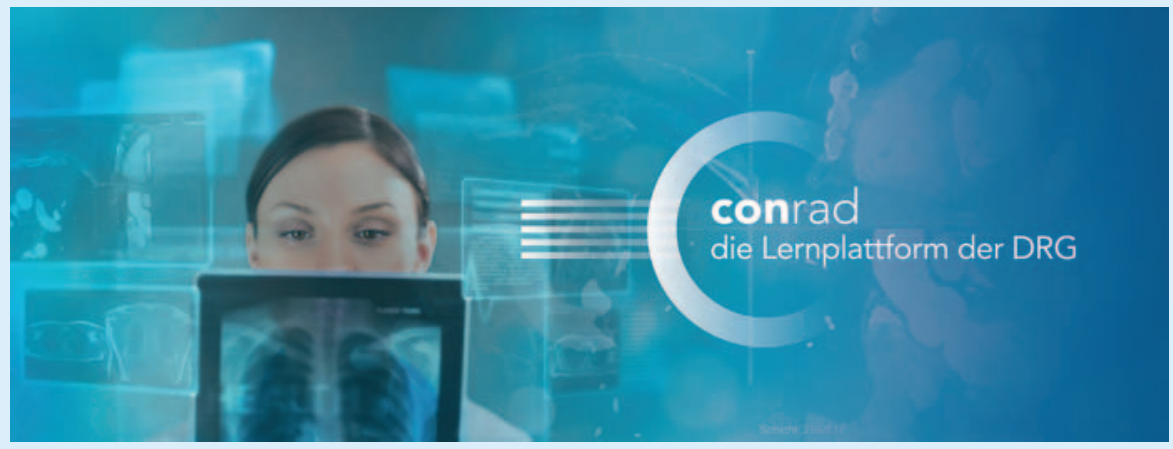

Die digitale Lernplattform der DRG bietet Lernmodule für Radiologinnen und Radiologen. (c) Deutsche Röntgengesellschaft. 